\title{
Resultados del bloqueo epidural caudal en pacientes laboralmente activos con dolor lumbar
}

\author{
Micaela Besse, José F. Baigorria, José A. Rosado Pardo, Ricardo Baldasarre, Leandro Ambrosini, \\ Aníbal J. Sarotto \\ Servicio de Ortopedia y Traumatología, Sanatorio "Victorio Franchín”, Ciudad Autónoma de Buenos Aires, Argentina
}

\begin{abstract}
RESUMEN
Introducción: El dolor lumbar es una de las entidades categorizadas por la Organización Mundial de la Salud como enfermedades prioritarias y una de las principales causas de ausentismo laboral. El bloqueo caudal epidural es una técnica anestésica utilizada para tratar el dolor lumbar de diversos orígenes. Materiales y Métodos: Se realizó un estudio observacional descriptivo retrospectivo en pacientes sometidos a bloqueo caudal guiado por radioscopia, entre el 1 de enero de 2017 y el 31 de enero de 2019. Se excluyó a los pacientes con bloqueo caudal previo, con antecedente de cirugía de columna lumbar y a quienes abandonaron el seguimiento. Se registraron variables relacionadas con el paciente y los resultados del procedimiento (dolor según la escala analógica visual, intervención quirúrgica posterior, actividad laboral posterior y licencia médica solicitada). Resultados: Se incluyó a 38 pacientes (media de la edad 43 años [rango 27-62]). La mediana de dolor a los tres meses fue de 3,5 (rango 2-6), con una diferencia de 4,5 (RIC de -2,7 a -7) respecto del puntaje basal. Estas diferencias fueron estadísticamente significativas ( $p$ $<0,001)$. El $55,3 \%$ retornó al trabajo al primer mes, y el $52,4 \%$ de ellos $(n=11)$ solo requirió una semana de licencia. Conclusiones: Los valores del dolor a corto plazo luego del procedimiento se modificaron de forma estadísticamente significativa respecto de los valores basales. El promedio de reinserción laboral al mes fue del 55,3\% ( $n=21)$ y el 76,2\% de ellos $(n=16)$ lo hizo a las dos semanas.
\end{abstract}

Palabras clave: Inyección epidural caudal; bloqueo caudal epidural; días laborales perdidos; dolor lumbar; retorno al trabajo. Nivel de Evidencia: IV

\section{Caudal Epidural Block Outcomes in Economically Active Patients With Low Back Pain}

\section{ABSTRACT}

Introduction: Low back pain is a very common health problem worldwide and a major cause of disability, affecting performance at work and general well-being. It is included in the priority diseases list of the World Health Organization (WHO). Low back pain is one of the leading causes of work absence worldwide. The caudal epidural block is a popular approach in managing lumbar pain conditions in adults. Materials and Methods: An observational, descriptive, retrospective study was conducted in our institution, from January 2017 to January 2019, on patients who had undergone caudal epidural block for low back pain. We excluded patients with a history of previous epidural blocks, lumbar surgery, or who were lost to follow up. We collected patient demographic and clinical data (including age, sex, etc.) and procedure outcomes (pain score, surgery performed, lost working days, and return to work activity). Results: 38 patients were included. Mean age was 43 (27-62) years old. Mean pain scores 3 months after treatment were $3.5(2-6)$ with a difference of 4.5 (IQR -2.7 - -7) from baseline values. This was statistically significant. $55.3 \%(n=21)$ of patients returned to active duty in the first month; it is important to note that $52.4 \%$ of those $(n=11)$ did it after the first week. Conclusions: The improvement of pain scores after the procedures was statistically significant. The mean return to work at the end of the first month was $55.3 \%$, and $76.2 \%$ of those $(n=16)$ did it after the first two weeks.

Key words: Caudal epidural injection; caudal block; missing working days; low back pain; return-to-work.

Level of Evidence: IV

Recibido el 25-3-2021. Aceptado luego de la evaluación el 4-5-2021 • Dra. MICAELA BESSE • m.besse @ hotmail.com.ar https://orcid.org/0000-0002-4388-1384 Cómo citar este artículo: Besse M, Baigorria JF, Rosado Pardo JA, Baldasarre R, Ambrosini L, Sarotto AJ. Resultados del bloqueo epidural caudal en pacientes laboralmente activos con dolor lumbar. Rev Asoc Argent Ortop Traumatol 2021;86(6):763-770. https://doi.org/10.15417/issn. 1852-7434.2021.86.6.1339 


\section{INTRODUCCIÓN}

El dolor lumbar es una de las entidades categorizadas por la Organización Mundial de la Salud como enfermedades prioritarias, ya que es un problema de salud en el mundo y afecta la calidad de vida y el desempeño laboral. La prevalencia en adultos ronda el $70 \%$ y es la primera causa de ausentismo laboral en gran parte del mundo. El impacto socioeconómico de esta entidad provocó un auge en el manejo y tratamiento adecuados y oportunos para mejorar la calidad de vida agilizando el retorno a la actividad laboral. ${ }^{1,2}$

Los bloqueos caudales epidurales fueron descritos, por primera vez, en 1901, como una técnica a ciegas guiada por referencias anatómicas, con altas tasas de fracasos, para tratar diversas causas de dolor lumbar. Recién, en 1940, resurge este procedimiento cuando Higson y cols. describen su uso en anestesia para el trabajo de parto y más tarde con el estudio de Dawkins que refleja un bajo porcentaje de complicaciones. En la actualidad, es una técnica anestésica ampliamente difundida en niños y en adultos para el manejo del dolor lumbar no quirúrgico, de diversos orígenes, pese a que sus resultados son controvertidos..$^{3-7}$

En el mundo, está aumentando la incidencia del dolor lumbar crónico y ante el gran impacto socioeconómico tanto individual como gubernamental que ocasiona, han cobrado importancia las técnicas terapéuticas no quirúrgicas. Entre ellas, el bloqueo caudal es una de las más difundidas, ya que alcanzan los niveles lumbares bajos, uno de los principales sitios de la enfermedad, con un bajo porcentaje de complicaciones.

Se ha demostrado el efecto a corto plazo de la aplicación de corticoides; en cambio, no está tan claro su efecto a largo plazo (luego de las 12 semanas) y la bibliografía médica refleja las controversias..$^{7-11}$

El objetivo de este estudio es comunicar la reinserción laboral de pacientes con dolor lumbar sometidos a bloqueo epidural caudal. Los objetivos secundarios son reportar la mejoría del dolor a corto plazo después del procedimiento y los resultados obtenidos en distintos cuadros.

\section{MATERIALES Y MÉTODOS}

\section{Diseño}

Se llevó a cabo un estudio observacional descriptivo retrospectivo en el Sanatorio "Victorio Franchín" de la Ciudad Autónoma de Buenos Aires, Argentina, entre el 1 de enero de 2017 y el 31 de enero de 2019. El Sanatorio es una institución dependiente de la obra social de la construcción (OSPECON).

\section{Consideraciones éticas}

Este estudio fue aprobado por el Comité de Ética e Investigación de la institución. Dado su carácter retrospectivo, no se solicitó el consentimiento informado de los participantes y los datos filiatorios se preservaron mediante su codificación en una base de datos con acceso exclusivo de los investigadores.

\section{Criterios de elegibilidad}

Se incluyó a todos los pacientes sometidos a un bloqueo caudal guiado por radioscopia que ingresaron en el período de estudio. Se excluyó a aquellos casos con bloqueo caudal previo, con antecedente de cirugía de columna lumbar y a quienes abandonaron el seguimiento.

\section{Recolección de datos}

La información fue organizada en una base de datos desarrollada por los mismos investigadores a partir de las historias clínicas de los pacientes. La información faltante se recolectó mediante el interrogatorio durante las visitas de seguimiento.

Se registraron variables relacionadas con el paciente (edad, sexo, etc.) y los resultados del procedimiento (dolor según la escala analógica visual, intervención quirúrgica posterior, actividad laboral posterior y licencia médica solicitada).

\section{Procedimiento}

Los procedimientos se llevaron a cabo en el quirófano del sanatorio, de forma ambulatoria, con acceso venoso periférico y monitoreo, y estuvieron a cargo del equipo de columna del Servicio de Ortopedia y Traumatología del Sanatorio "Victorio Franchín". Todo el personal de salud participante utiliza elementos de radioprotección (chaleco plomado con protección tiroidea).

Se coloca a los pacientes en decúbito prono, con un realce a nivel pélvico. Se realiza la antisepsia de la piel con solución con yodopovidona y se colocan campos estériles. Mediante la palpación de reparos anatómicos, se loca- 
liza el hiato sacro (identificando las espinas ilíacas posterosuperiores, el cóccix y los cuernos sacros), se infiltra la piel con lidocaína al $2 \%$ y se coloca una aguja de $22 \mathrm{G}$ en el hiato sacro. Se utiliza una guía radioscópica para controlar la posición y se progresa con la aguja hasta la tercera vértebra sacra. Una vez que se controla la posición adecuada de la aguja, se introducen $10 \mathrm{cc}$ de solución fisiológica, seguidos de una mezcla de 2 cc de lidocaína sin epinefrina al $2 \%$ y $2 \mathrm{mg} / \mathrm{ml}$ de triamcinolona.

Se indicó reposo laboral por $72 \mathrm{~h}$ luego del procedimiento y los controles se realizaron a las tres semanas y a los tres meses.

\section{Análisis estadístico}

Las variables categóricas se expresan como número absoluto de presentación y porcentaje. Las variables continuas que asumieron una distribución normal se expresan como media y desviación estándar (DE) o como mediana y rango intercuartílico (RIC). Para verificar la distribución de la muestra se utilizó la prueba de Shapiro-Wilk.

Para comparar el dolor antes de la intervención y después se utilizó la prueba t de Student para muestras relacionadas o la prueba de rangos con signo de Wilcoxon para muestras relacionadas, según correspondiera. Las comparaciones entre grupos independientes de variables se realizaron con la prueba $t$ de Student para muestras independientes o la prueba U de Mann-Whitney, según lo apropiado.

Los datos se analizaron con el programa IBM SPSS Macintosh, versión 24.0 (IBM Corp., Armonk, NY, EE.UU.).

\section{RESULTADOS}

Se indicó el procedimiento a 61 pacientes y se realizó en 56 casos. Diez pacientes fueron excluidos, porque cursaban el posoperatorio de una cirugía lumbar o tenían antecedente de bloqueo, cuatro abandonaron el seguimiento y cuatro no pudieron ser incluidos por datos incompletos en la historia clínica. Tres fueron retirados por intercurrencias y dos se negaron a participar. Por lo tanto, se incluyeron 38 pacientes en el análisis. La media de edad de la muestra fue de 43 años (mín. 27, máx. 62). Doce (31,6\%) eran mujeres. El resto de las características de la muestra se detallan en la Tabla 1.

Tabla 1. Características de la muestra

\begin{tabular}{|l|c|}
\hline Variables & $\mathrm{n}=38$ \\
\hline Sexo femenino, n (\%) & $12(31,6)$ \\
\hline Edad, media (DE), años & $43.3(10.2)$ \\
\hline Motivo de consulta, n (\%) & \\
Lumbalgia & $15(39,5)$ \\
\hline Lumbociatalgia & $23(60,5)$ \\
\hline Diagnóstico, n (\%) & \\
Canal estrecho lumbar & $6(15,8)$ \\
\hline Discopatía central & $22(57,9)$ \\
\hline Discopatía posterolateral & $5(13,2)$ \\
\hline Más de una afección & $5(13,2)$ \\
\hline Nivel afectado, n (\%) & \\
L3/L4 & $1(2,6)$ \\
\hline L4/L5 & $7(18,4)$ \\
\hline L5/S1 & $14(36,8)$ \\
\hline L3/L4/L5/S1 & $16(42,1)$ \\
\hline Dolor basal, mediana (RIC) & $9(8-9)$ \\
\hline
\end{tabular}

RIC = rango intercuartílico, DE = desviación estándar. 
La mediana de dolor evaluado a los tres meses fue 3,5 puntos (rango 2-6), con una diferencia de 4,5 puntos (RIC de -2,7 a -7) en relación con el puntaje basal. Estas diferencias resultaron estadísticamente significativas ( $<<0,001)$ (Figura 1).

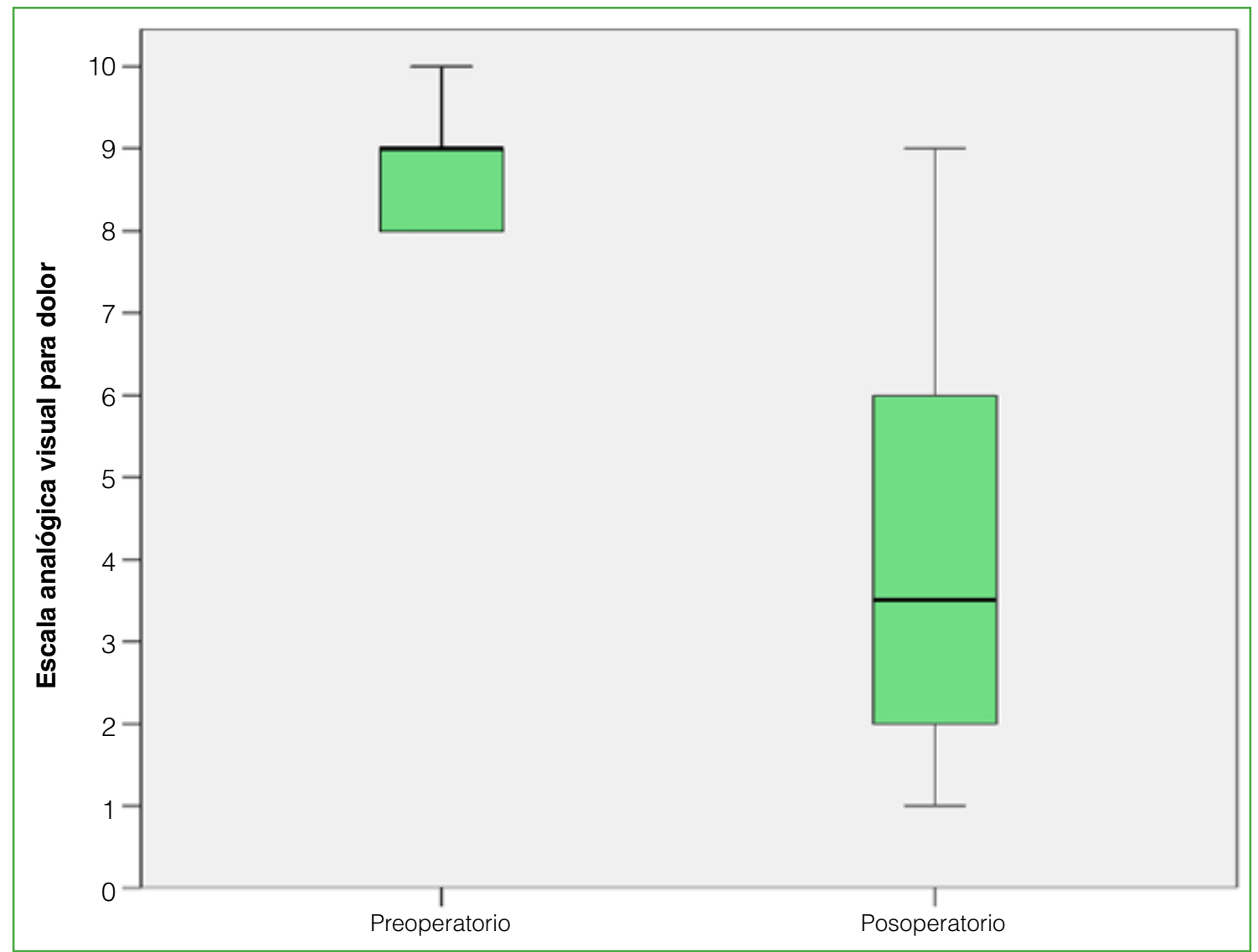

Figura 1. Valores de dolor preoperatorio y a los tres meses del procedimiento.

Los pacientes con discopatía posterolateral tuvieron un cambio de 7 puntos (RIC 1,5-7) en el dolor comparados con aquellos que presentaban discopatía central y un canal estrecho lumbar quienes presentaron una disminución de 5 (RIC 2-7) y 4,5 (RIC 2,25-5,5) puntos, respectivamente. El cambio fue de 4 puntos (RIC 3,5-6,5) en los pacientes con más de un diagnóstico (Tabla 2).

Con respecto al motivo de consulta, el cambio en el dolor fue de 5 (RIC 4-7) puntos en los pacientes con lumbalgia y de 4 (RIC 2-7) en aquellos con lumbociatalgia. Estas diferencias no resultaron estadísticamente significativas $(\mathrm{p}=0,18)$.

Cinco pacientes $(13,1 \%)$ requirieron cirugía. Dos fueron intervenidos dentro del mes.

Veintiún $(55,3 \%)$ pacientes requirieron una licencia laboral de hasta cuatro semanas. De ellos, $11(52,4 \%)$ requirieron una semana; cinco $(23,8 \%)$, hasta dos semanas, tres $(14,3 \%)$ y dos $(9,5 \%), 4$ semanas (Figura 2$)$.

Veintidós $(57,9 \%)$ mantuvieron su trabajo, ocho $(21,1 \%)$ lo cambiaron y ocho $(21,1 \%)$ lo perdieron. 
Tabla 2. Cambios en el dolor según el diagnóstico

\begin{tabular}{|l|c|c|c|c|}
\hline Diagnóstico & n & Dolor preoperatorio & Dolor posoperatorio & Diferencia \\
\hline Canal estrecho lumbar & 6 & $8,5(8-9)$ & $4(2,7-6,5)$ & $4,5(2,2-5,5)$ \\
\hline Discopatía central & 22 & $9(8-9)$ & $3(2-6)$ & $5(2-7)$ \\
\hline Discopatía posterolateral & 5 & $9(9-10)$ & $3(2-8)$ & $7(1,5-7)$ \\
\hline Más de una afección & 5 & $10(8-10)$ & $4(2,5-6,5)$ & $4(3,5-6,5)$ \\
\hline
\end{tabular}

Valores expresados en mediana y rango intercuartílico.

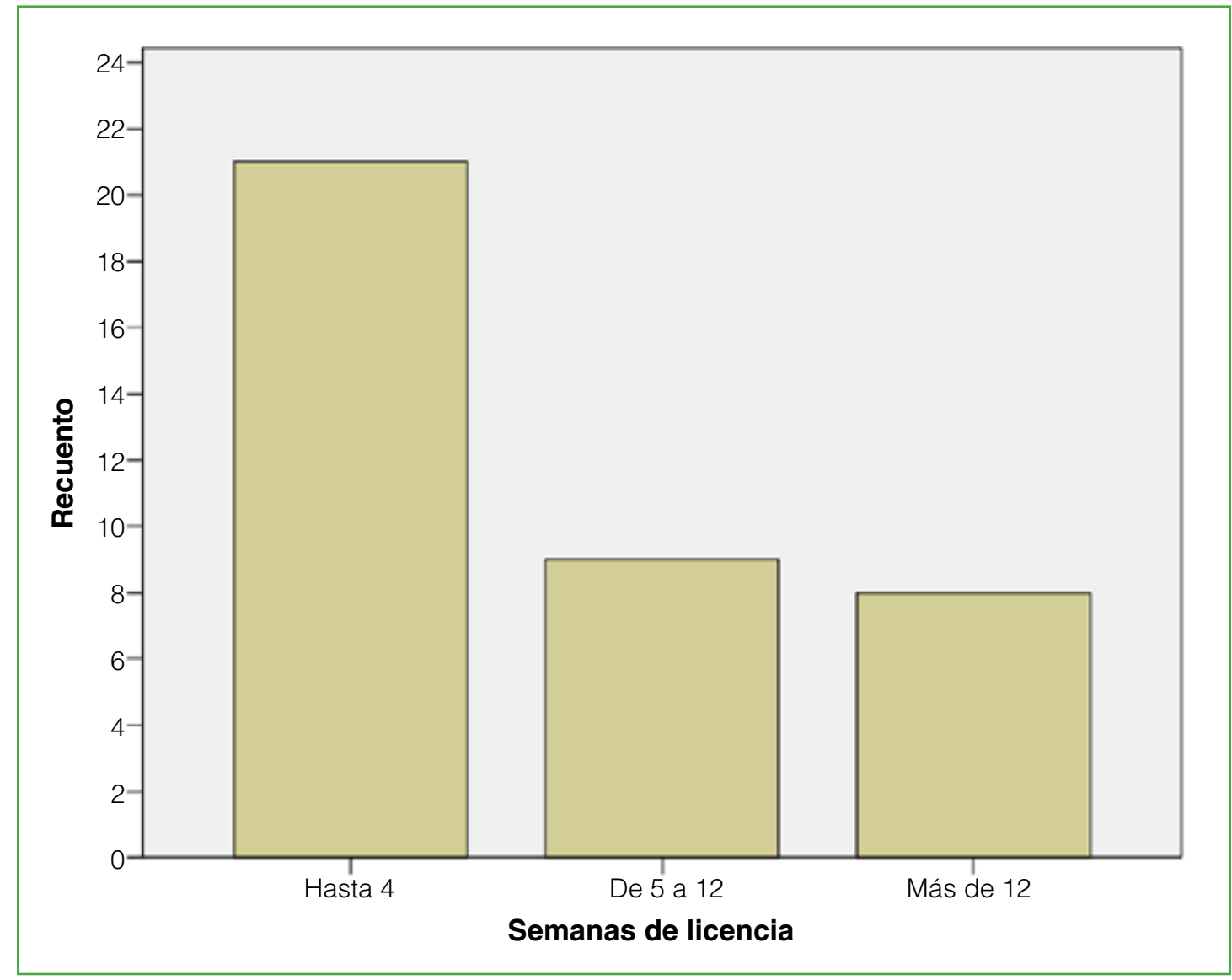

Figura 2. Semanas de licencia por paciente.

\section{DISCUSIÓN}

$\mathrm{Al}$ canal epidural lumbar se puede acceder por vía interlaminar, foraminal o caudal. En el primer caso, se coloca la medicación en el sitio donde se asume la fuente del dolor; la vía foraminal requiere menor volumen, ya que es más específica aún. El acceso caudal es considerado la vía más fácil y de mejor acceso, con mínimos riesgos de lesión del saco dural, pese a que requiere mayores volúmenes, y tiene la ventaja de alcanzar distintos niveles afectados. $^{7-9}$

El anestésico local utilizado fue lidocaína sin epinefrina. En cuanto al corticoide, hay publicaciones sobre el uso de diversos corticoides, principalmente triamcinolona, betametasona y metilprednisolona. En este estudio, administramos triamcinolona. 
Los elementos para asegurar el correcto posicionamiento de la aguja en el conducto sacro son: 1) reparos anatómicos y 2) guía radioscópica o ecográfica. El reparo anatómico es el hiato sacro, formado por la disrafia del arco posterior de la quinta, y menos frecuentemente, la cuarta vértebra sacra; su límite lateral son los cuernos sacros formados por los remanentes de las articulares inferiores elongadas hacia inferior. Como estos reparos pueden no palparse (cuando miden $<3 \mathrm{~mm}$ ), sobre todo en personas obesas, otro reparo es un triángulo equilátero formado por las crestas ilíacas posterosuperiores y el hiato sacro. ${ }^{11-14}$

Diversas publicaciones demuestran que, en niños, el acceso a ciegas tiene altas tasas de éxito (96\%); sin embargo, en adultos, disminuye (68-75\%) incluso en manos experimentadas. Por este motivo, actualmente, se prefiere la guía con radioscopia o la guía ecográfica, que surge como opción para disminuir la radiación utilizada y como elemento de fácil acceso. ${ }^{11-13}$ En nuestra práctica habitual, empleamos la guía radioscópica.

El mecanismo de acción del corticoide y anestésico local administrados por esta vía no está del todo dilucidado. Se cree que el bloqueo de la transmisión neural alcanzado altera o interrumpe los aferentes nociceptivos y las vías de transmisión del dolor. Los corticoides disminuyen la inflamación a través de la inhibición de la síntesis o la liberación de mediadores proinflamatorios y con un efecto anestésico local reversible. El anestésico local provoca una mejoría sintomática a corto plazo por el bloqueo de la descarga nociceptiva, el bloqueo del transporte axonal y del arco reflejo simpático, lo que produce, a su vez, un efecto a largo plazo, probablemente por una modificación plástica de la transmisión de la información aferente proveniente de los nociceptores..$^{8,9}$

Se han publicado resultados contradictorios sobre la eficacia de este tratamiento para el dolor lumbar de diversos orígenes. La primera revisión sistemática fue realizada por Kepes y Duncalf, en 1985, y estos autores no encontraron justificación para realizar este procedimiento. En 1986, el grupo de Benzon obtuvo buenos resultados con la administración de corticoides para los cuadros de dolor lumbar, principalmente aquellos que cursan con irritación nerviosa. Desde ese momento, se llevaron a cabo múltiples estudios, y algunos de ellos con resultados contradictorios. ${ }^{9}$

En una revisión bibliográfica, Conn y cols. hallaron evidencia de nivel I para la eficacia del bloqueo caudal, a corto y largo plazo (punto de corte, 6 meses) para la lumbalgia secundaria a: discopatía lumbar, radiculopatía y dolor discogénico; no así para aquellas secundarias a canal estrecho o síndrome poslaminectomía, donde la evidencia disminuye a nivel II-III. ${ }^{8}$

Asimismo, Parr y cols. encontraron buena evidencia en la eficacia del tratamiento para las lumbalgias secundarias a discopatía y radiculitis, no así para el dolor axial, la estenosis del canal y el síndrome poslaminectomía. ${ }^{9}$ En concordancia con estos autores, hemos obtenido peores resultados en los pacientes con conducto lumbar estrecho. En una revisión sistemática reciente, Oliveira y cols. hallaron evidencia de calidad moderada para la eficacia de las inyecciones epidurales de corticoide en pacientes con lumbociatalgia; sin embargo, el análisis realizado incluyó publicaciones con diversos accesos al espacio epidural (foraminal, caudal e interlaminar) y diversos corticoides (prednisolona, triamcinolona y metilprednisolona). ${ }^{7}$

La Organización Mundial de la Salud define el dolor lumbar como la entidad que lidera el gasto económico relacionado con la salud, en los Estados Unidos, asciende a más de 100 billones de dólares anuales y es una de las principales causas de ausentismo laboral y la causa más común de discapacidad laboral. Afecta principalmente a los trabajadores de entre 35 y 55 años. ${ }^{1,14,15}$

En la revisión sistemática de Wynne-Jones y cols., la tasa de retorno laboral fue del $68 \%$ al mes. En nuestro estudio, el 55,3\% reanudó el trabajo al primer mes, y el 52,4\% de ellos $(\mathrm{n}=11)$ solo requirió una semana de licencia.

Los días perdidos anualizados por esta causa se calculan en 149 millones, solo en los Estados Unidos de Norteamérica. Lo que ocasiona un gasto no solo en el tratamiento de la entidad, sino también en la disminución de la producción (gastos primario y secundario).

En nuestro país, los días perdidos en el ámbito de la construcción están entre los más altos de la economía nacional. En el Reporte estadístico del Ministerio de Trabajo, Empleo y Seguridad Social, ya en 2007, alcanzaban los 4080 días perdidos por cada mil trabajadores y fue la segunda causa de ausentismo laboral. En las estadísticas de 2017, el promedio de jornadas laborales perdidas, en el sector de la construcción fue de 32,4. ${ }^{16-18}$

Los bloqueos caudales representan en el medio de la obra social de la construcción un valor calculado que se aproxima a los $\$ 35.000$ (valor calculado según tabla de hora quirófano), si bien es un costo significativo, intentamos reducir los días perdidos por inactividad laboral a dos semanas, como en el $42 \%(\mathrm{n}=16)$ de los pacientes de nuestro estudio. Esto podría ejercer un impacto significativo en el costo por días perdidos (al descender el promedio aproximado de 32 a 15 días) (datos de costos de la administración sanatorial). 
Las limitaciones de este estudio son su diseño retrospectivo, el escaso número de la muestra y que esta pertenece a una población de actividad laboral de carga, lo que puede condicionar algunos de los resultados.

Destacamos como fortaleza del estudio que los procedimientos fueron realizados por el Equipo de Columna, bajo guía radioscópica. Esto podría estar relacionado con el buen resultado a las dos semanas, al disminuir la tasa de errores en el procedimiento.

\section{CONCLUSIONES}

El tasa promedio de reinserción laboral al mes fue del 55,3\% $(n=21)$, se destaca que el 76,2\% de ellos $(n=16)$ se reincorporó a las dos semanas. Los valores del dolor a los tres meses del procedimiento se modificaron, de forma estadísticamente significativa, respecto a los valores basales. Los pacientes con conducto lumbar estrecho fueron los que obtuvieron los peores resultados, aquellos con discopatías posterocentrales y posterolaterales tuvieron resultados similares.

Conflicto de intereses: Los autores no declaran conflictos de intereses.

ORCID de J. F. Baigorria: https://orcid.org/0000-0002-3453-2246 ORCID de J. A. Rosado Pardo: https://orcid.org/0000-0001-8467-3453 ORCID de R. Baldasarre: https://orcid.org/0000-0002-8426-9204
ORCID de L. Ambrosini: https://orcid.org/0000-0003-1378-4660 ORCID de A. J. Sarotto: https://orcid.org/0000-0002-2199-5524

\section{BIBLIOGRAFÍA}

1. WHO. Priority Medicines for Europe and the World 2013 Update. Disponible en: https://www.who.int/medicines/areas/priority_medicines/Ch6_24LBP.pdf

2. Duthey B. Update on 2004 Background Paper, BP 6.24 Low back pain. Priority Medicines for Europe and the World. “A Public Health Approach to Innovation”. WHO. Disponible en: https://www.who.int/medicines/areas/priority_medicines/BP6_24LBP.pdf

3. Cortiñas Sáenz M, Iglesias Cerrillo JA, Cano Navarro G, Salmerón Cerezuela J, Quirante Pizarro JA, Carricondo Martínez MI, et al. Bloqueo caudal en dolor crónico lumbar. ¿Es necesario el apoyo radiológico para disminuir los fallos de la técnica? Rev Soc Esp Dolor 2012;19(4):174-80. Disponible en: http://scielo.isciii.es/scielo.php?script=sci_arttext\&pid=S1134-80462012000400003

4. Higson RA, Edwuars WB. An analysis of the first ten thousand confinements managed with continuous caudal analgesia with a report of the authors, first one thousand cases. JAMA 1943;123(9):538-46. https://doi.org/10.1001/jama.1943.02840440020006

5. Dieng P, Diouf E, Diene JF. Caudal epidural anesthesia in 70 children. Dakar Med 1994;39:95 7. PMID: 7493530

6. Dawkins CJM. An analysis of the complications of extradural and caudal block. Anaesthesia 1969;24(4):554-63. https//doi.org/10.1111/j.1365-2044.1969.tb02909.x

7. Oliveira CB, Maher CG, Ferreira ML, Hancock MJ, Oliveira VC, McLachlan AJ, et al. Epidural corticosteroid injections for sciatica. Spine (Phila Pa 1976) 2020;45(21):E1405-E1415. https://doi.org/10.1097/BRS.0000000000003651

8. Conn A, MD, Buenaventura RM, Datta S, Abdi S, Diwan S. Systematic review of caudal epidural injections in the management of chronic low back pain. Pain Physician 2009;12:109-35. PMID: 19165299

9. Parr AT, Manchikanti L, Hameed H, Conn A, Manchikanti KN, Benyamin RM, et al. Caudal epidural injections in the management of chronic low back pain: a systematic appraisal of the literature. Pain Physician 2012; 15(3):E159-E198. PMID: 22622911

10. Nandi J, Chowdhery A. RCT to determine effectiveness of caudal epidural steroid injection in lumbosacral sciatica. J Clin Diagn Res 2017;11(2):RC04-RC08. https://doi.org/10.7860/JCDR/2017/21905.9392

11. Kao SC, Lin CS. Caudal epidural block: an updated review of anatomy and techniques. Biomed Res Int 2017;2017: 9217145. https://doi.org/10.1155/2017/9217145 
12. Waldman SD. Caudal epidural nerve block: prone position. En: Waldman SD (ed.). Atlas of interventional pain management. $2^{\text {nd }}$ ed. Philadelphia: Saunders; 2004:380-92.

13. Stitz MY, Sommer HM. Accuracy of blind versus fluoroscopically guided caudal epidural injection. Spine (Phila PA 1976) 1999;24(13):1371-6. https://doi.org/10.1097/00007632-199907010-00016

14. Aggarwal A, Aggarwal A, Harjeet, Sahni D. Morphometry of sacral hiatus and its clinical relevance in caudal epidural block. Surg Radiol Anat 2009;31(10):793-800. https://doi.org/10.1007/s00276-009-0529-4

15. Low Back Pain Fact Sheet. NIH. NINDS, Publication date December 2014. Publication No. 15-5161. Disponible en: https://www.ninds.nih.gov/Disorders/Patient-Caregiver-Education/Fact-Sheets/Low-Back-Pain-Fact-Sheet

16. Wynne-Jones G, Cowen J, Jordan JL, Uthman O, Main CJ, Glozier N, et al. Absence from work and return to work in people with back pain: a systematic review and meta-analysis. Occup Environ Med 2014;71(6):448-56. https://doi.org/10.1136/oemed-2013-101571

17. Estadísticas sobre Accidentabilidad Laboral. Ministerio de Trabajo, Empleo y Seguridad Social, Superintendencia de Riesgos del Trabajo 2007. Disponible en: www.infoleg.gob.ar/basehome/actos_gobierno/actosdegobierno7-12-2009-3.htm

18. Departamento de Estudios y Estadísticas. Informe Anual de Accidentabilidad Laboral 2017. Ciudad Autónoma de Buenos Aires: Superintendencia de Riesgos del Trabajo. Disponible en:

https://www.argentina.gob.ar/sites/default/files/informe_anual_de_accidentabilidad_laboral_-_ano_2017.pdf 